\title{
Aspects of Unparticle Physics
}

\author{
Arvind Rajaraman \\ Department of Physics and Astronomy, University of California, Irvine, CA 92697, USA
}

\begin{abstract}
We review some theoretical and experimental issues in unparticle physics, focusing mainly on collider signatures.
Keywords: Unparticles

PACS: 12 .60.Fr, 13.25.Jx, 14.80.Cp
\end{abstract}

\section{INTRODUCTION AND MOTIVATION}

It is widely believed that the Standard Model is not a complete theory of particle physics, and that there is a new physics sector coupled to the Standard Model, which addresses various problems of the Standard Model such as the hierarchy problem and the identity of dark matter. A priori, this new physics can be of several basic forms. It can be weakly coupled, like supersymmetric theories. Another class of theories is technicolor, where the new physics is strongly coupled.

Logically, there is a third option; the new physics could be conformal (or scale-invariant). This is the idea of unparticles. The idea (and the name) was introduced by Georgi in [1] (for a recent review of unparticles, and further references to the literature, see [2]). As we shall discuss here, unparticles may provide many interesting and unexpected signals at the upcoming Large Hadron Collider (LHC).

\section{THE UNPARTICLE THEORY}

To discuss the signals of unparticles, we need

- A description of the hidden sector (the CFT)

- A form for the interactions between the CFT and the SM.

\section{The hidden sector}

There are many possible examples of conformal theories, e.g. Banks-Zaks theories [3] and supersymmetric QCD in the conformal window[4]. We will take a very simple model for the conformal sector by focusing on a few operators of the theory rather than the full complexity. In fact, we will assume that the only coupling to the conformal sector is through a single operator $O_{U}$ ( this operator $O_{U}$ can be a scalar, vector, fermion or a higher spin object.)
Now the propagator for this operator is fixed by conformal invariance. For example, the propagator for scalar unparticles in an exactly conformal theory is fixed to be [5, 6]

$$
T\left\langle 0\left|O_{U}(x) O_{U}^{\dagger}(0)\right| 0\right\rangle=i B_{d}\left(p^{2}\right)^{d-2}
$$

where $B_{d}$ is a constant, and $d$ is the dimension of the operator $O_{U}$. There are similar expressions for vector and fermion operators.

The momentum space propagator can also be written as a dispersion integral [5, 7]

$T\left\langle 0\left|O_{U}(x) O_{U}^{\dagger}(0)\right| 0\right\rangle=i \frac{A_{d}}{2 \pi} \int_{0}^{\infty} d M^{2} \frac{\rho\left(M^{2}\right)}{p^{2}-\mu^{2}-M^{2}+i \varepsilon}$

with $\rho\left(M^{2}\right)=\left(M^{2}\right)^{d-2}$. This representation shows that the propagator can be understood as a sum over resonances where the resonance masses are continuously distributed. In particular, there is no mass gap.

\section{Constraints on the dimension}

The dimension is constrained by the unitarity of the conformal algebra 1 . The unitarity imposes lower bounds on the dimension [8]

- Scalar operators: $d \geq 1$

- Fermion operators: $d \geq 3 / 2$

- Gauge invariant vector operators: $d \geq 3$

On the other hand we can also find upper bounds on the dimension. Since $\rho\left(M^{2}\right)=\left(M^{2}\right)^{d-2}$. we see that for $\mathrm{d}>2$, the theory is ultraviolet sensitive; small changes at high energies radically alter the propagator. This has several consequences. In particular, we find singular behavior in many situations with $\mathrm{d}>2$ e.g. the energy density at finite temperature and some cross-sections are propor-

\footnotetext{
1 These limits can be avoided if the theory is scale-invariant, but not conformal.
} 
tional to (2-d) [9]. These imply that we should restrict ourselves to $d<2$ ( $d<5 / 2$ for fermions).

Combining this with the lower bounds above, we find that vector and higher spin operators are problematic; accordingly we will focus on scalar operators.

\section{Standard Model Interactions}

The unparticle operator is coupled to the Standard Model by terms of the form $O_{U} O_{S M}$. The only constraint on these couplings is dimensional analysis. There are therefore a huge number of possible couplings. Schematically, we can have a Lagrangian

$L_{i n t}=\Lambda^{2-d} O_{U} H^{2}+c_{\psi} \Lambda^{1-d} O_{U} \bar{\psi} \psi+c_{F} \Lambda^{-d} O_{U} F^{2}$

For every quark pair, lepton pair and gauge field, we have an independent coupling. These couplings are unconstrained by theory.

\section{THE UNPARTICLE MASS GAP}

We now begin the study of the experimental signals of unparticles.

As we have mentioned above, exact scale invariance precludes a mass gap for unparticles. In an exactly scaleinvariant theory, unparticles would mediate long range forces. They would also contribute to precision experiments like $(g-2)_{\mu}$ through loop effects. Since they are effectively massless, this can be a big effect. Indeed, as shown in several papers (see e.g. [6, 10, 11, 12, 13]), the constraints are strong enough to rule out any possibility of seeing unparticles at the LHC.

We now argue that in fact interactions induce a mass gap for unparticles. This removes all these low energy constraints.

This happens due to the Higgs couplings $\Lambda^{2-d} \mathrm{OH}^{2}$. This coupling is a relevant operator; it becomes important at low energies. In particular it breaks the conformal invariance of the low-energy theory [14]. This is clear from the fact that once the Higgs field gets a vev $H=v+h$, we get linear terms as well as Higgsunparticle mixing. This then introduces a scale $\mu$ with $\mu^{4-d}=\Lambda^{2-d} v^{2}$ and breaks scale invariance in the hidden sector.

The introduction of the scale modifies the density of resonances $\rho\left(M^{2}\right)$. The modification is model dependent [15], but generically, we find that a mass gap is introduced i. e. $\rho\left(M^{2}\right)=0$ for $M^{2} \ll m^{2}$. Also, for high energies, the density is unchanged: $\rho\left(M^{2}\right)=\left(M^{2}\right)^{d-2}$ for $M^{2} \gg m^{2}$.

Since $\Lambda \sim v \sim 100 \mathrm{GeV}$, we find that in the absence of fine tuning, the mass gap is at least a few $\mathrm{GeV}$.

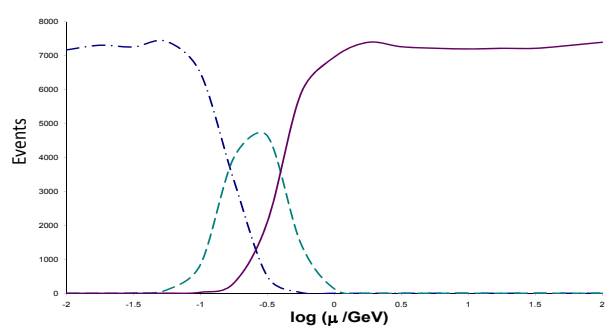

FIGURE 1. Number of events with $10 \mathrm{fb}^{-1}$ of LHC data as a function of $\mu$. The solid (red) line corresponds to the number of prompt events, dot-dashed (blue) corresponds to the number of monojet events, and dashed (green) is the number of delayed events. We have taken $d=1.1$ and $\Lambda=10000 \mathrm{GeV}$.

This immediately implies that there are no long range forces from unparticle exchange. Precision constraints (say from $\left.(g-2)_{\mu}\right)$ essentially disappear.

This further implies that low energy experiments are not sensitive to unparticles: unparticles are best probed at colliders. We therefore turn to collider signals of unparticles.

\section{COLLIDER SIGNATURES: UNPARTICLE DECAYS}

For definiteness, we will henceforth consider a situation where the unparticle couples primarily to gauge bosons

$$
L_{i n t}=\frac{O_{U} F_{\mu v} F^{\mu v}}{\Lambda_{F}^{d}}+\frac{O_{U} G_{\mu v} G^{\mu v}}{\Lambda_{G}^{d}}
$$

where $F_{\mu \nu}, G_{\mu \nu}$ are the electromagnetic and color field strengths respectively, and $\Lambda_{F}, \Lambda_{G}$ are scales parametrizing the couplings. We will take for simplicity $\Lambda_{F} \sim \Lambda_{G}=$ $\Lambda$.

Unparticles can now be produced at hadron colliders through processes like $g g \rightarrow g O_{U}$. If the unparticle does not decay we get monojets (and more generally, missing energy signals.) In fact, as we now show, unparticles can decay to SM particles. This will modify this set of signals.

The basic point is that loop corrections can modify the unparticle propagator. Resumming the contributions, we obtain the full propagator

$$
T\left\langle 0\left|O_{U}(x) O_{U}^{\dagger}(0)\right| 0\right\rangle=\frac{i B_{d}}{\left(p^{2}-\mu^{2}\right)^{2-d}-B_{d} \Sigma\left(p^{2}\right)}
$$

where the loop diagram is $-i \Sigma\left(p^{2}\right)$. The propagator can develop an imaginary piece and this leads to unparticle decay. The width is proportional to the imaginary part of $B_{d} \Sigma\left(p^{2}\right)$. 


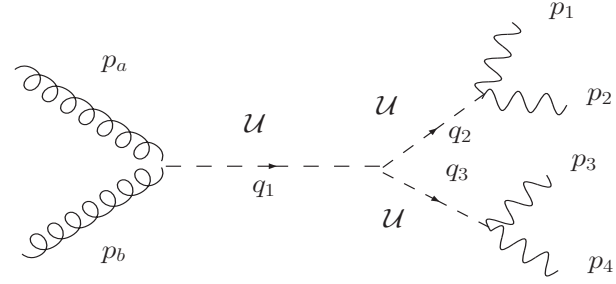

FIGURE 2. The process $g g \rightarrow \gamma \gamma \gamma \gamma$ mediated by unparticle self-interactions.

If unparticles are produced at colliders, they can themselves decay back to Standard Model particles. Depending on the lifetime, we have different signals. If the lifetime is short, we have prompt decays. If the lifetime is very long, we get monojets and missing energy signals. Most interesting is the intermediate situation, where we can get delayed events and displaced vertices.

We calculate the number of each type of event, with 10 $\mathrm{fb}$ of LHC data. We take $\mathrm{d}=1.1, \Lambda=10 \mathrm{TeV}$. We require the gluon jet to have energy $>100 \mathrm{GeV}$. We shall further assume that the detector is $1 \mathrm{~m}$ in size, and that delays of 100ps can be measured [16].

Our results are shown in Fig 1 For $\mu>10 \mathrm{GeV}$, we only have prompt events. There are a significant number of monojets only if $\mu<100 \mathrm{MeV}$, which requires some fine tuning as discussed earlier. Interestingly, in an intermediate range $(\mu \sim 1 \mathrm{GeV})$, there are a large number of delayed events. These could have a striking signal at the LHC.

\section{UNPARTICLE SELF-INTERACTIONS}

We now look at signals from unparticle self-interactions.

The three-point interactions of operators are fixed by conformal invariance up to an overall constant

$$
\left\langle 0\left|O(x) O(y) O^{\dagger}(0)\right| 0\right\rangle=\frac{C}{|x-y|^{d}|x|^{d}|y|^{d}},
$$

Assume again that the unparticle couples to gluons and photons as in eq. (4). At the Tevatron and LHC we can now have four-photon processes as shown in Fig 2

The rate of these processes is controlled by the constant $C$ in the three point-function, which is not constrained by theory. Now we can use experimental input to constrain the allowed value of $C$. This analysis was performed in [17], where it was found that the primary bound comes from the nonobservation of excess fourphoton events at the Tevatron. Assuming that $C$ is as large as the experimental limit, we can predict the rate of these processes at the LHC and ILC. These rates are shown in Fig 3 . We find that there are huge rates for these

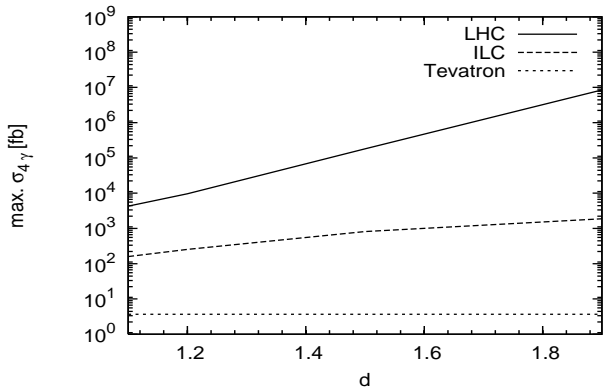

FIGURE 3. Cross-sections at the LHC and ILC for the process $g g \rightarrow \gamma \gamma \gamma \gamma$.

processes, which could again provide striking signals of unparticles.

To conclude, we have shown that unparticles are an interesting alternative model for the hidden sector. In these models, we can obtain many unique and striking signals at colliders such as delayed events, displaced vertices and multiphoton processes. Further work on unparticles may well lead to other new and interesting signals that would otherwise have been missed.

\section{ACKNOWLEDGMENTS}

This work is supported in part by NSF Grants No. PHY0354993 and PHY-0653656.

\section{REFERENCES}

1. H. Georgi, Phys. Rev. Lett. 98 (2007) 221601 |arXiv:hep-ph/0703260].

2. Kingman Cheung, Wai-Yee Keung, Tzu-Chiang Yuan, arXiv:0809.0995 1 [hep-ph].

3. See, T. Banks and A. Zaks, Nucl. Phys. B 196, 189 (1982).

4. See, K. A. Intriligator and N. Seiberg, Nucl. Phys. Proc. Suppl. 45BC, 1 (1996) |arXiv:hep-th/9509066|.

5. H. Georgi, Phys. Lett. B 650 (2007) 275 [arXiv:0704.2457 [hep-ph]].

6. K. Cheung, W. Y. Keung and T. C. Yuan, Phys. Rev. Lett. 99, 051803 (2007) [arXiv:0704.2588 [hep-ph]].

7. M. A. Stephanov, Phys. Rev. D 76, 035008 (2007) arXiv:0705.3049[hep-ph]].

8. G. Mack, Commun. Math. Phys. 55, 1 (1977).

9. G. Cacciapaglia, G. Marandella and J. Terning, JHEP 0801, 070 (2008) [arXiv:0708.0005 [hep-ph]].

10. M. Luo and G. Zhu, Phys. Lett. B 659, 341 (2008) |arXiv:0704.3532 [hep-ph]].

11. C. H. Chen and C. Q. Geng, Phys. Rev. D 76, 115003 (2007) |arXiv:0705.0689 [hep-ph]].

12. G. J. Ding and M. L. Yan, Phys. Rev. D 76, 075005 (2007) arXiv:0705.0794 [hep-ph]].

13. Y. Liao, Phys. Rev. D 76, 056006 (2007) arXiv:0705.0837 [hep-ph]]. 
14. P. J. Fox, A. Rajaraman and Y. Shirman, Phys. Rev. D 76, 075004 (2007) |arXiv:0705.3092 [hep-ph]].

15. A. Delgado, J. R. Espinosa and M. Quiros, JHEP 0710, 094 (2007) [arXiv:0707.4309 [hep-ph]].

16. S. Vigano and A. De Min, "Detection methods for long lived particles at the LHC," Prepared for IFAE 2006 (in Italian), Pavia, Italy, 19-21 Apr 2006 See also www.pv.infn.it/ ifae2006/talks/NuovaFisica/Vigano.ppt

17. J. L. Feng, A. Rajaraman and H. Tu, Phys. Rev. D 77, 075007 (2008) |arXiv:0801.1534 [hep-ph]]. 